\title{
REDUCED ORDER MODELS FOR NONLINEAR DYNAMIC ANALYSIS WITH APPLICATION TO A FAN BLADE
}

\author{
Mikel Balmaseda* \\ DMAS, ONERA, Université Paris-Saclay \\ F-92322 Châtillon, France \\ Université de Lyon, CNRS INSA Lyon \\ LaMCoS UMR 5259 \\ F-69621Villeurbanne, France \\ Email: mikel.balmaseda@onera.fr
}

\author{
G. Jacquet-Richardet \\ Université de Lyon, CNRS INSA Lyon \\ LaMCoS UMR 5259 \\ F-69621Villeurbanne, France \\ Email: georges.jacquet@insa-lyon.fr
}

\author{
A. Placzek \\ DAAA, ONERA, Université Paris-Saclay \\ F-92322 Châtillon, France \\ Email: antoine.placzek@onera.fr
}

\author{
D.-M. Tran \\ DMAS, ONERA, Université Paris-Saclay \\ F-92322 Châtillon, France \\ Email: tran@onera.fr
}

\begin{abstract}
In the present work reduced order models (ROM) that are independent from the full order finite element models (FOM) considering geometrical non linearities are developed and applied to the dynamic study of a fan. The structure is considered to present nonlinear vibrations around the pre-stressed equilibrium induced by rotation enhancing the classical linearised approach. The reduced nonlinear forces are represented by a polynomial expansion obtained by the Stiffness Evaluation Procedure (STEP) and then corrected by means of a Proper Orthogonal Decomposition (POD) that filters the full order nonlinear forces (StepC ROM). The Linear Normal Modes (LNM) and Craig-Bampton (C-B) type reduced basis are considered here. The
\end{abstract}

${ }^{*}$ Corresponding author 
latter are parametrised with respect to the rotating velocity. The periodic solutions obtained with the StepC ROM are in good agreement with the solutions of the FOM and are more accurate than the linearised ROM solutions and the STEP ROM. The proposed StepC ROM provides the best compromise between accuracy and time consumption of the ROM.

\section{INTRODUCTION}

The large displacements observed in the dynamic response of rotating blades in turbomachinery, for instance when mistuning occurs, highlight the necessity to accurately predict the geometrically nonlinear behaviour of these structures in order to improve their design (weight, dimensions...). The tendency to create more slender and flexible structures increases the nonlinear behaviour of these components. Furthermore, as repetitive high fidelity nonlinear finite element computations are expensive to carry out, reduced order models that provide good compromise between accuracy and time performances are a useful tool for the designer.

The nonlinear forces in the reduced order model are generally computed by methods that need to perform full order model (FOM) computations during the solution process. The Discrete Empirical Interpolation (DEIM) [1] provides an interpolation of the nonlinear term at a reduced cost in a Galerkin projection context by computing nonlinear forces in a given number of previously chosen interpolation points. Hyper-reduction $[2,3]$ evaluates the nonlinear behaviour law of the structure in a reduced domain. The piecewise linearisation [4] is an alternative technique that eases the system matrix computation issues. An efficient approach to nonlinear structural analysis was carried by [5] representing the internal forces by a third order polynomial formulation as a function of displacements. This method is known as the Stiffness Evaluation Procedure (STEP). The stiffness coefficients of the polynomial representation are obtained by a series of static results obtained with the full order finite element model. As an extension to STEP method "non intrusive" reduced order models independant of the FOM have been reviewed by [6] and validated for the prediction of fatigue, nonlinear stochastic computations [7], post-buckling analyses [8] and complex structures. The Element-wise Stiffness Evaluation Procedure (E-STEP) [9] generalizes the STEP to optimisation problems enabling the parametrisation of the stiffness evaluation procedure.

In the framework of rotating structures, a comparative study between several models of a rotating cantilever beam in terms of accuracy and validity is presented in [10]. These models are mainly used in the study of helicopter and turbo-machinery blades [11], modelisations of slender beams or thin shells [12] and fluid-structure interactions [13]. A finite element formulation of the rotating problem is presented in [14] and the necessity to develop 3D finite element models for the study of rotordynamics is highlighted in [15]. In the mechanics of jointed structures the use of component mode synthesis $[16,17]$ is widely applied to implement Iwan hysteresis $[18,19]$ contact models. Petrov [20] proposed an analytical formulation for the vectors of contact forces and the stiffness matrix of the nonlinear friction contact interface for the analysis of multi-harmonic vibrations in the frequency domain. Then a numericalexperimental study of the forced responses for mistuned bladed was carried out [21].

In the classical finite element formulation for geometrically nonlinear rotating structures [22] the vibrations around the pre-stressed static equilibrium are considered as small and linear. Here we asume that those vibrations are nonlinear enhancing the classical linearised 
small displacements hypothesis considering that large displacement may occur when the structure vibrates around the static equilibrium state. Thus, as an extension to [23], an autonomous geometrically nonlinear reduced order model for the study of dynamic solutions of complex rotating structures is developed. Within the scope of implementing contact type nonlinearities in the future, a Craig-Bampton type reduced basis is constructed. To compute the nonlinear forces in the reduced order model the stiffness evaluation procedure method (STEP) is applied. The nonlinear forces are corrected by means of a filtering of the nonlinear forces so that only their components belonging to the subspace spanned by the Proper Orthogonal Decomposition (POD) basis of the nonlinear forces are kept. This POD basis is obtained by performing a Singular Value Decomposition (SVD) to a set of nonlinear force snapshots. The proposed ROM is then applied to the dynamic study of a 3D blade obtaining encouraging results for LNM and Craig-Bampton reduced basis with parametrisation.

The remainder is structured as follows: first, the theoretical concepts of rotating structure dynamics are developed. Second, the construction of the geometrically nonlinear reduced order model with the nonlinear POD based correction is presented. Third, the developed reduced order models are evaluated for a fan type of blade. Finally, some concluding remarks are drawn.

\section{DYNAMICS OF ROTATING STRUCTURES}

The finite element discretisation to study the dynamics of rotating structures is developed in [14]. The discretised equation of motion of a high fidelity model of a rotating structure is presented in Eqn. (1) considering the rotating frame of reference. The latter model, also called Full Order Model (FOM) provides accurate results at a computationally expensive cost. In the following, we consider that the rotating velocity has a constant modulus and that the axis of rotation does not vary. In order to magnify the influence of geometric nonlinearities, the gyroscopic and the Coriolis effects are neglected with respect to the centrifugal ones.

$$
\mathbf{M} \ddot{\mathbf{u}}_{p}+\mathbf{C} \dot{\mathbf{u}}_{p}+\mathbf{K}_{c}(\Omega) \mathbf{u}_{p}+\mathbf{g}\left(\mathbf{u}_{p}\right)=\mathbf{f}_{e}(t)+\mathbf{f}_{e i}(\Omega)+\mathbf{f}_{c}\left(\mathbf{u}_{p}, \dot{\mathbf{u}}_{p}\right)
$$

where $\mathbf{M}$ is the mass matrix, $\mathbf{C}$ is the viscous damping matrix, $\mathbf{K}_{c}(\Omega)$ is the centrifugal softening matrix, $\mathbf{g}\left(\mathbf{u}_{p}\right)$ is the nonlinear internal forces vector, $\mathbf{f}_{e}(t)$ is the external forces vector, $\mathbf{f}_{e i}(\Omega)$ is the centrifugal forces vector and $\mathbf{f}_{c}\left(\mathbf{u}_{p}, \dot{\mathbf{u}}_{p}\right)$ is the nonlinear forces due to friction contact.

To reduce the complexity of Eqn. (1) the physical displacements are defined as the sum of an initial pre-stressed static equilibrium state, $\mathbf{u}_{s}$, induced by rotation and the relative displacements, $\mathbf{u}$, around the pre-stressed static equilibrium state as $\mathbf{u}_{p}=\mathbf{u}_{s}+\mathbf{u}$ where the static equilibrium state is obtained by solving the nonlinear system of Eqn. (2). 


$$
\mathbf{K}_{c}(\Omega) \mathbf{u}_{s}+\mathbf{g}\left(\mathbf{u}_{\mathrm{s}}\right)=\mathbf{f}_{e i}(\Omega) .
$$

Depending on the characteristics of the studied contact nonlinearity (tip shroud, rub...) contact effects should be considered in Eqn. (2). In the classical approach [22] the static state of equilibrium due to the rotating velocity $\Omega$ is obtained by considering the geometrically nonlinear effects (large displacements) and the dynamic state around the static state is linearised by means of the tangent stiffness matrix. In the approach we introduce here, not only the static state is considered with a nonlinear behaviour but also the dynamic vibrations around the static state, keeping the nonlinear behaviour of the internal forces. The latter is represented by a purely nonlinear forces term $\mathbf{g}_{n l}(\mathbf{u})$ as shown in Eqn. (3). In the following, the classical linearised approach is referred to as the "Linear FOM/ROM". The equation of motion in terms of the relative displacements is presented in [24].

$$
\mathbf{M} \ddot{\mathbf{u}}+\mathbf{C} \dot{\mathbf{u}}+\mathbf{K}(\Omega) \mathbf{u}+\mathbf{g}_{n l}(\mathbf{u})=\mathbf{f}_{e}(t)+\mathbf{f}_{c}\left(\mathbf{u}+\mathbf{u}_{s}, \dot{\mathbf{u}}\right)
$$

where $\mathbf{K}=\mathbf{K}_{c}(\Omega)+\mathbf{K}_{e}+\mathbf{K}_{n l}\left(\mathbf{u}_{s}\right)$ is the stiffness matrix composed of the linear elastic stiffness matrix $\mathbf{K}_{e}$, of the centrifugal softening matrix and of the nonlinear part of the tangent matrix which includes the geometrical pre-stressed stiffness matrix $\mathbf{K}_{g}\left(\mathbf{u}_{s}\right)$.

The FOM has an expensive time cost for large number of computations (i.e. design, control...). In order to significantly reduce the computational time in exchange of an acceptable loss in precision reduced order model techniques are implemented in the following.

\section{GEOMETRICALLY NONLINEAR ROM}

In the present section the size of the FOM is reduced by means of projection-based model order reduction techniques. When the structure presents a nonlinear behaviour due to large displacements or due to contact/friction effects, the reduced order model should integrate these nonlinearities into its construction.

\section{Reduced Basis}

The reduced order models (ROMs) constructed by projection on a reduced basis are widely developed in literature. These methods differentiate between an expensive "OFFline" stage where the projection basis are computed and an efficient "ONline" stage where the problem is solved. One of these methods whose "OFFline" stage is computationally efficient and is widely developed in commercial 
finite element codes is the basis formed by the Linear Normal Modes (LNM) of the structure. However, the latter technique is inefficient to solve contact problems as none of the generalised coordinates represents a physical displacement. In order to develop ROMs that solve interface problems such as contact, we use component mode synthesis techniques that provide appropriate reduced bases as some degrees of freedom (d.o.f.) of interest on the interface are kept in the generalised coordinates.

Here we implement the Craig-Bampton method [16] to combine it with the POD based nonlinear forces correction [24] within the scope of implementing a ROM that considers geometrical and contact nonlinearities in the future.

The physical displacements for the Craig-Bampton method are composed by the inner d.o.f., $\mathbf{u}_{i}$, and by the boundary interface d.o.f., $\mathbf{u}_{b}$. With this method stiffness and mass matrices are partitioned with respect to inner and boundary d.o.f.

$$
\mathbf{K}(\Omega)=\left[\begin{array}{ll}
\mathbf{K}_{i i} & \mathbf{K}_{i b} \\
\mathbf{K}_{b i} & \mathbf{K}_{b b}
\end{array}\right] \quad, \quad \mathbf{M}=\left[\begin{array}{ll}
\mathbf{M}_{i i} & \mathbf{M}_{i b} \\
\mathbf{M}_{b i} & \mathbf{M}_{b b}
\end{array}\right] .
$$

The same partition is applicable to all the elements in the equation of motion of the structure, Eqn. (1).

Then, as for any projection based reduced order model, the relative physical displacements of the FOM are approximated as a linear combination between the reduction basis and the generalised displacements as shown in Eqn. (5).

$$
\mathbf{u}=\mathbf{Q q}=\Phi_{C B}\left\{\begin{array}{c}
\mathbf{q}_{i} \\
\mathbf{u}_{b}
\end{array}\right\}=\left[\Phi_{c} \Psi_{c}\right]\left\{\begin{array}{c}
\mathbf{q}_{i} \\
\mathbf{u}_{b}
\end{array}\right\}
$$

In the Craig-Bampton method the projection basis $\mathbf{Q}=\Phi_{C B}$ is composed of $r_{c}$ fixed interface linear normal modes $\Phi_{c}$ and $r_{b}$ constraint modes $\Psi_{c}$, while the generalised coordinates $\mathbf{q}$ are composed of the modal coordinates $\mathbf{q}_{i}$ and the boundary interface d.o.f. $\mathbf{u}_{b}$. The number of vectors in the projection basis $\mathbf{Q}$ is then $r=r_{c}+r_{b}$.

Fixed Interface Linear Normal Modes. The stiffness of a rotating structure depends on the rotating velocity and on the nonlinear static deformation of the pre-stressed equilibrium state. Thus, the natural frequencies and the modes of the structure depend on the rotating velocity. To compute the fixed interface linear normal modes, the pre-stressed equilibrium state is obtained by solving a nonlinear problem that only considers the effect of the centrifugal forces induced by rotation. Then, as shown in Eqn. (6) the linearised LNM are computed by considering that the structure is clamped at the boundary interface d.o.f. 


$$
\mathbf{K}(\Omega) \Phi_{c}=\mathbf{M} \Phi_{c} \omega^{2} \quad,\left.\quad \Phi_{c}\right|_{u_{b}}=\mathbf{0}
$$

The linear normal modes basis is formed by truncating the solution basis to the first $r_{c}$ modes, $\Phi_{c}=\left[\Phi_{c_{1}}, \ldots, \Phi_{c_{r_{c}}}\right]$. The associated natural frequencies of the fixed interface structure are $\omega=\operatorname{diag}\left[\omega_{1}, \ldots, \omega_{r_{c}}\right]$

Constraint Modes. The static deformation solution to unitary displacements at boundary d.o.f of the structure represent the constraint modes as shown in Eqn. (7).

$$
\mathbf{K}(\Omega)\left\{\begin{array}{c}
\Psi_{c_{i}} \\
\mathbf{I}
\end{array}\right\}=\left[\begin{array}{cc}
\mathbf{K}_{i i} & \mathbf{K}_{i b} \\
\mathbf{K}_{b i} & \mathbf{K}_{b b}
\end{array}\right]\left\{\begin{array}{c}
\Psi_{c_{i}} \\
\mathbf{I}
\end{array}\right\}=\left\{\begin{array}{c}
\mathbf{0} \\
\mathbf{R}
\end{array}\right\}
$$

The constraint modes $\Psi_{c}$ are then obtained from Eqn. (7) as:

$$
\Psi_{c}=\left\{\begin{array}{c}
-\mathbf{K}_{i i}^{-1} \mathbf{K}_{i b} \\
\mathbf{I}
\end{array}\right\}
$$

Craig-Bampton Reduced Order Basis. The Craig-Bampton reduced basis presented in Eqn. (5) is formed by the fixed interface linear normal modes and the constraint modes of the structure.

$$
\Phi_{C B}=\left[\Phi_{c} \Psi_{c}\right]=\left[\begin{array}{cc}
\Phi_{c_{i}} & \Psi_{c_{i}} \\
\mathbf{0} & \mathbf{I}
\end{array}\right]
$$




\section{Construction Of The Reduced Order Model}

Once the reduction basis is computed, the reduced order model is obtained by a Galerkin projection and the displacements approximation defined in Eqn. (5). Then, the reduced order equation of motion of the structure with dimension $r$ is defined as:

$$
\tilde{\mathbf{M}} \ddot{\mathbf{q}}+\tilde{\mathbf{C}} \dot{\mathbf{q}}+\tilde{\mathbf{K}} \mathbf{q}+\tilde{\mathbf{g}}_{n l}(\mathbf{q})=\tilde{\mathbf{f}}_{e}(t)+\tilde{\mathbf{f}}_{c}(\mathbf{q}, \dot{\mathbf{q}})
$$

with $\tilde{\mathbf{M}}=\Phi_{C B}^{T} \mathbf{M} \Phi_{C B}, \tilde{\mathbf{C}}=\Phi_{C B}^{T} \mathbf{C} \Phi_{C B}, \tilde{\mathbf{K}}=\Phi_{C B}^{T} \mathbf{K} \Phi_{C B}, \tilde{\mathbf{g}}_{n l}(\mathbf{q})=\Phi_{C B}^{T} \mathbf{g}_{l l}(\mathbf{u}), \tilde{\mathbf{f}}_{e}(t)=\Phi_{C B}^{T} \mathbf{f}_{e}(\mathbf{t})$ and $\tilde{\mathbf{f}}_{c}(\mathbf{q}, \dot{\mathbf{q}})=\Phi_{C B}^{T} \mathbf{f}_{c}(\mathbf{u}, \dot{\mathbf{u}})$.

By partitioning Eqn. (10), the contact forces vector only applies to the boundary d.o.f. as shown in Eqn. (11). Thus, contact in the reduced order model is solved with any technique available in the FOM [25-27].

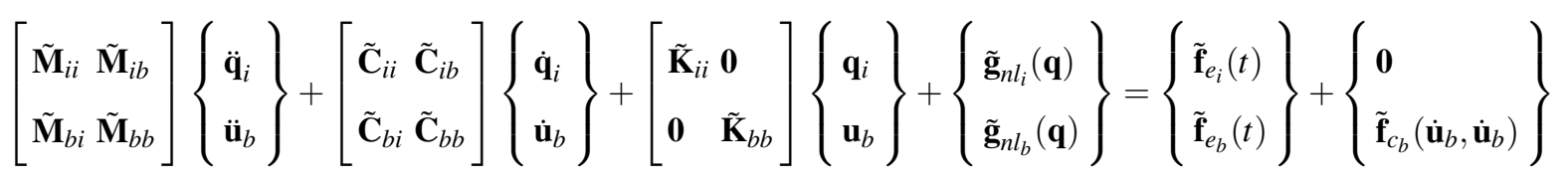

Parametrization of the ROM The proposed ROM is obtained by solving Eqn . (6) for each considered rotating velocities. Thus, reconstructions of the ROM is required for analysing the response at different rotating speeds and could limit the analysis for loadings that depend on the rotating velocity, i.e. engine order excitations. To reduce the latter limitation, a parametrization of the ROM is carried out as shown in [28,29].

The parametrised reduced order basis is obtained by carrying out a Singular Value Decomposition (SVD) of the reduced basis at three precomputed rotating velocities, $\left\{\mathbf{Q}_{\Omega=p 0}, \mathbf{Q}_{\Omega=p 0+\Delta p}, \mathbf{Q}_{\Omega=p 0+2 \Delta p}\right\}$ where $p 0, p 0+\Delta p$ and $p 0+2 \Delta p$ represent the rotating velocities used for interpolation.

Furthermore, the stiffness matrix is defined as a function of the rotating velocity by a quadratic interpolation:

$$
\mathbf{K}(\Omega)=\mathbf{K}(p 0)+\left.\frac{\partial \mathbf{K}}{\partial \Omega}\right|_{\Omega=p 0}(\Omega-p 0)+\left.\frac{1}{2} \frac{\partial^{2} \mathbf{K}}{\partial \Omega^{2}}\right|_{\Omega=p 0}(\Omega-p 0)^{2}
$$

where the derivatives are identified by means of finite order differences. 


\section{Nonlinear Forces In The Reduced Space}

Many different methods exist to compute the generalised nonlinear forces in the reduced space: the inflation method has a simple implementation in commercial codes, however, for every nonlinear force evaluation, the nonlinear forces are computed by the FOM and then projected to obtain the generalised forces of the ROM. This method produces computationally inefficient ROMs that depend on the size of the FOM. POD/DEIM method uses a second basis for the nonlinear term. Then the nonlinear forces are evaluated at DEIM interpolation points and the nonlinear term is approximated through collocation in the nonlinear POD basis. Hyper-reduction technique is adapted to problems involving internal variables. The constitutive equations are solved in a reduced domain of the structure and the internal variable are extrapolated by using POD vectors related to the internal variables. In order to avoid carrying out FOM computations within the "ONline" stage, hereinafter, we use the STiffness Evaluation Procedure (STEP) that approximate the nonlinear forces as a third degree polynomial. Furthermore, to improve the solution of the nonlinear forces projection, we introduce a POD based correction for nonlinear forces combined with a Craig-Bampton basis.

STEP Polynomial Approximation. Equation (12) defines the generalised nonlinear forces as a third degree polynomial approximation.

$$
\tilde{g}_{n l}^{p}\left(q_{1}, \ldots, q_{r}\right)=\sum_{i=1}^{r} \sum_{j=i}^{r} A_{i j}^{p} q_{i} q_{j}+\sum_{i=1}^{r} \sum_{j=i}^{r} \sum_{m=j}^{r} B_{i j m}^{p} q_{i} q_{j} q_{m}
$$

The stiffness coeffients are evaluated during the "OFFline" stage carrying out $\left(r^{3}+6 r^{2}+5 r\right) / 6$ nonlinear forces static evaluations where $r$ corresponds to the number of modes in the reduction basis. An example on how to compute the polynomial coefficients is presented in [23].

POD Based Correction For Nonlinear Forces. The projection of nonlinear forces in slender structures by a linear basis (LNM or Craig-Bampton) to obtain the generalised forces in the ROM creates spourious artifacts [30] in the time response that induce lower amplitude displacement with higher harmonics. Thus, to correct such behaviour induced by projection a filtering process of FOM nonlinear forces is carried out here. The filtering is obtained by a nonlinear forces basis computed previously during the "OFFline" stage.

As shown in Eqn. (13), the nonlinear forces are collected from a given number of snapshots that represent a set of characteristic displacements in the response. Then, for each snapshot the associated nonlinear forces are evaluated, 


$$
\mathbf{A}=\left[\mathbf{g}_{n l}\left(\mathbf{u}_{1}\right), \cdots, \mathbf{g}_{n l}\left(\mathbf{u}_{m}\right)\right]
$$

and the nonlinear basis is obtained by implementing a truncated Singular Value Decomposition (SVD) [31].

$$
\mathbf{A}=\mathbf{U} \Sigma \mathbf{V}^{T} \approx \mathbf{U}_{r_{f}} \Sigma_{r_{f}} \mathbf{V}_{r_{f}}^{T}
$$

The nonlinear basis consists in the truncation to $r_{f}$ modes in the resulting left singular vectors of the SVD basis, $\Phi_{f}=\mathbf{U}_{r_{f}}$ verifying $\Phi_{f}^{T} \Phi_{f}=\mathbf{I}$.

Once the nonlinear forces basis is constructed, as defined in Eqn. (15), the nonlinear forces in the FOM are approximated as a linear combination between the nonlinear forces basis and the approximated force coordinates.

$$
\mathbf{g}_{n l}(\mathbf{u}) \approx \Phi_{f} \mathbf{q}_{n l}^{f}=\mathbf{g}_{n l}^{f}(\mathbf{u})
$$

where the force coordinates are computed by a least-squares approach. The approximation of the FOM nonlinear forces in the FOM space is computed by Eqn. (16).

$$
\mathbf{g}_{n l}^{f}(\mathbf{u})=\Phi_{f}\left(\Phi_{f}^{T} \Phi_{f}\right)^{-1} \Phi_{f}^{T} \mathbf{g}_{n l}(\mathbf{u})=\Phi_{f} \Phi_{f}^{T} \mathbf{g}_{n l}(\mathbf{u})
$$

Then, the generalised forces in the ROM space are, with $\mathbf{u}=\Phi_{C B} \mathbf{q}$ :

$$
\tilde{\mathbf{g}}_{n l}(\mathbf{q})=\Phi_{C B}^{T} \mathbf{g}_{n l}^{f}(\mathbf{u})=\Phi_{C B}^{T} \Phi_{f} \Phi_{f}^{T} \mathbf{g}_{n l}(\mathbf{u})=\mathbf{B}^{T} \mathbf{g}_{n l}(\mathbf{u})
$$

The proposed correction for nonlinear forces is a non intrusive technique as it only takes place in the projection of the nonlinear 


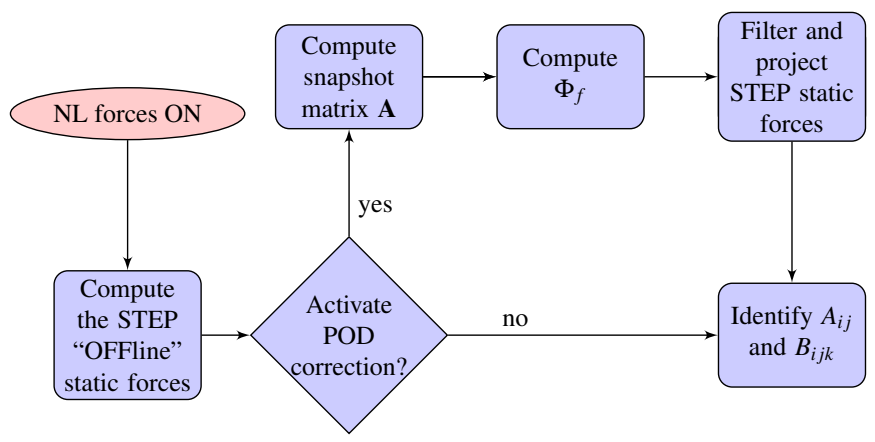

FIGURE 1. IDENTIFICATION OF NONLINEAR FORCES COEFFICIENTS BY STEP AND STEPC POD CORRECTION.

forces into the ROM space and does not require additional FOM computations once it is constructed.

Then, combining the STEP method and the proposed POD correction, the StepC ROM is constructed. Figure 1 represents the chart flow to identify the corrected stiffness coefficients, $A_{i j}$ and $B_{i j k}$. Then the nonlinear forces are computed by Eqn. (12).

\section{NUMERICAL APPLICATION}

The proposed ROMs are evaluated for the blade shown in Fig. 2, derived from the one designed in the frame of the activities devoted to the development of ONERA's NOVA configurations [32,33]. The finite element mesh is formed by 25872 hexahedral linear elements and 29681 nodes. The base surface of the blade is clamped and the external loadings are applied at every node of the top surface. The structure rotates at a nominal velocity $\Omega_{N}=4043 \mathrm{rpm}$ around the absolute $x$ axis. The material is a Titanium-Aluminium (TiAl) alloy with 117.5 GPa Young Modulus, 0.3 Poisson's Ratio, $4450 \mathrm{Kg} / \mathrm{m}^{3}$ density, $1 \%$ damping ratio and $828 \mathrm{MPa}$ Yield strength [34]. An inertial Rayleigh damping is considered to model the structural viscous damping, $\mathbf{C}=\beta_{m} \mathbf{M}=2 \xi \omega_{0} \mathbf{M}$. The time responses provided by the ROMs are studied at the leading edge node (control coordinate), highlighted in Fig. 2, as it is one of the regions where the greatest geometrically nonlinear effects are expected. The contact interface in Fig. 2, where contact could be implemented, corresponds to boundary interface d.o.f. of the Craig-Bampton method. This type of contact interface could model the interaction between the blade and the rubbing.

To study the periodic response under large displacements, induced for instance by mistuning or any other instability, the structure is excited at its first linearised natural frequency that corresponds to the first bending mode. Thus, the external forces frequency is obtained from the eigenvalue analysis of the linearised model from Eqn. (6) with free interfaces. The external harmonic force is applied at every node of the top surface in the axial direction as shown in Eqn. (18).

$$
\mathbf{f}_{e}(\mathrm{t})=\alpha_{f} \cdot \sin \left(\omega_{e} t\right) \cdot \vec{i}
$$




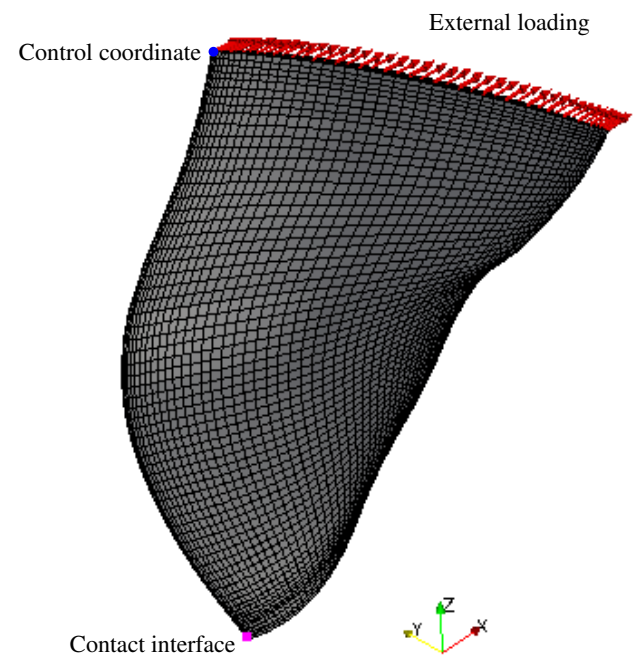

FIGURE 2. MESH OF THE BLADE WITH THE INTERFACE NODE HIGHLIGHTED.

Due to the nonlinear behaviour of the structure, the linearised ROM and the nonlinear FOM responses differ significantly as shown in Fig. 4 and 5, thus, the necessity to build a more accurate nonlinear ROM is highlighted.

Two types of parametrised reduced bases are considered in this application: Linear Normal Modes and Craig-Bampton. In order to favour the "OFFline" computational time of the nonlinear force coefficients the total number of modes is chosen to be $r=10$. In the case of Craig-Bampton basis, 7 fixed interface modes and 3 constraint modes are chosen (one for each d.o.f. of the studied interface node). For each basis type three different ROMs are studied. The linear ROMs do not take into consideration the purely nonlinear force term, the STEP ROMs consider the nonlinear term without any correction and the proposed StepC (Step with Correction) ROMs consider the nonlinear term with the POD correction developed previously. To avoid the construction of the projection basis at every rotating velocity, and to reduce the "OFFline" computational time, the reduced system is parametrised with respect to the rotating velocity.

The dynamic response of the structure is evaluated for three rotating velocities: for non rotating case, for a rotating velocity of $2000 \mathrm{rpm}$ and for $4043 \mathrm{rpm}$ rotating velocity where the last corresponds to the nominal rotating velocity $\Omega_{N}$ of the blade. The periodic solutions are computed with the HHT- $\alpha$ method for 100 excitation periods and 3000 time steps.

The parametric ROM is constructed by evaluating the reduced order basis and the stiffness matrix of the FOM at $0 \mathrm{rpm}, 2250 \mathrm{rpm}$ and $4500 \mathrm{rpm}$ rotating velocities. The reduced order basis are formed by the first 10 modes in order to keep a compromise between the "OFFline" computational cost and the accuracy of the ROMs.

\section{Convergence Analysis}

Figure 3 presents the convergence analysis for the STEP and StepC methods with respect to the number of modes that form the reduced LNM basis. The relative error is evaluated for the periodic regime as shown in Eqn. (19) for three different rotating velocities. The loading factor $\alpha_{f}$ is chosen such that nonlinear effects are observed. 


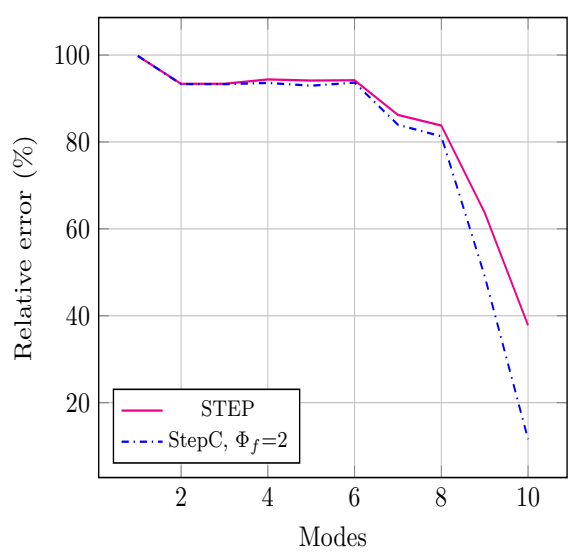

(a) $\Omega=0 \mathrm{rpm}, \alpha_{f}=0.5$.

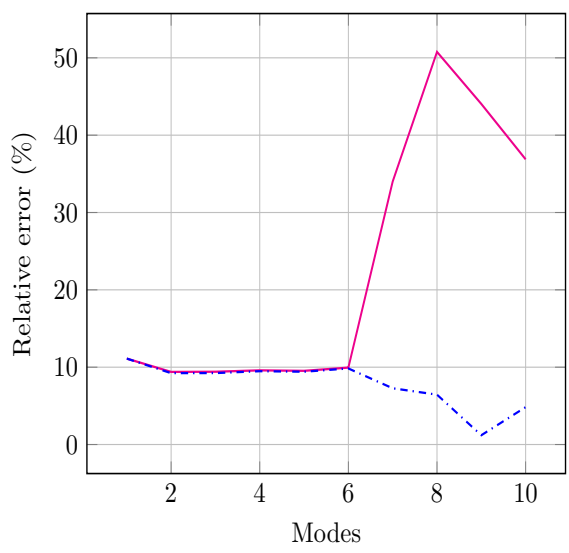

(b) $\Omega=2000 \mathrm{rpm}, \alpha_{f}=0.5$.

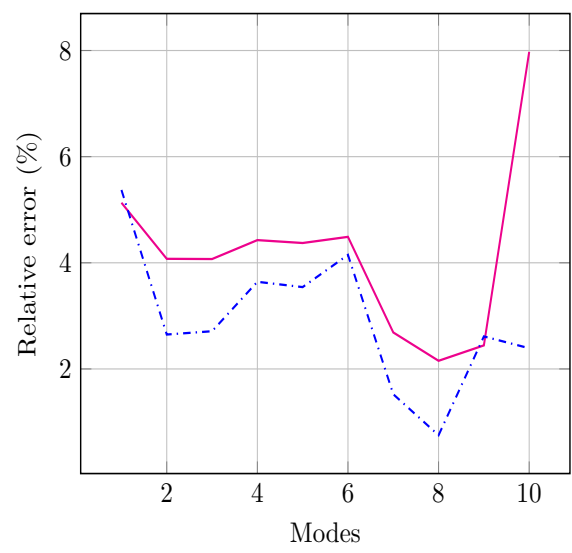

(c) $\Omega=4043 \mathrm{rpm}, \alpha_{f}=1.5$.

FIGURE 3. CONVERGENCE ANALYSIS FOR DIFFERENT NUMBER OF MODES FORMING THE REDUCED LNM BASIS.

$$
e_{C \text { Conv }}(\%)=\frac{\left|\max \left\{u_{R O M}(t)\right\}-\max \left\{u_{F O M}(t)\right\}\right|}{\max \left\{u_{R O M}(t)\right\}} \cdot 100 .
$$

It is observed that the correction does not influence the accuracy for a low number of modes and provides similar results to the STEP method. However, from the 6th mode of the basis the StepC correction with two nonlinear forces modes basis provide more accurate results for all the evaluated rotating velocities for a given basis size. Normally, when rotating velocity varies, the nonlinear basis $\Phi_{f}$ should be recomputed to adapt the ROM to the new velocity, however, it was observed that the nonlinear basis obtained at a given velocity can be used for another rotating velocity. The interest of using the same nonlinear forces basis for several rotating velocities is that the FOM forces, that are computationally very expensive to obtain, are not re-evaluated. For the convergence analysis, the nonlinear forces basis is formed by the first two modes in the resulting left singular vectors of the SVD computed by means of Eqn. (14), at a rotating velocity of $2000 \mathrm{rpm}$.

\section{Results}

We consider that nonlinear effects are significant when the difference between the linearised ROM and the nonlinear FOM periodic solutions differ by more than $10 \%$. For these cases, the cost of developing nonlinear ROMs is justified.

Figures 4 and 5 show the periodic solution of the blade at $0 \mathrm{rpm}, 2000 \mathrm{rpm}$ and $4043 \mathrm{rpm}$. The loading factor $\alpha_{f}$ is chosen to be $0.5 \mathrm{~N}$ for the first two rotating velocities and 1.5 for the last one in order to obtain a significant difference between the linearised and the nonlinear FOM solutions. The StepC nonlinear models provide more precise results than the linearised ROM and the STEP ROM for both reduced basis. It is observed that the solutions have a shift in time. This effect is highlighted when $\Omega$ differs from the rotating 


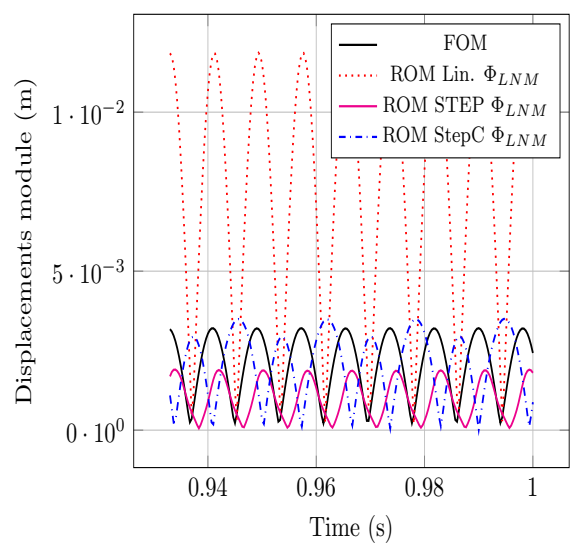

(a) $\Omega=0 \mathrm{rpm}, \alpha_{f}=0.5$.

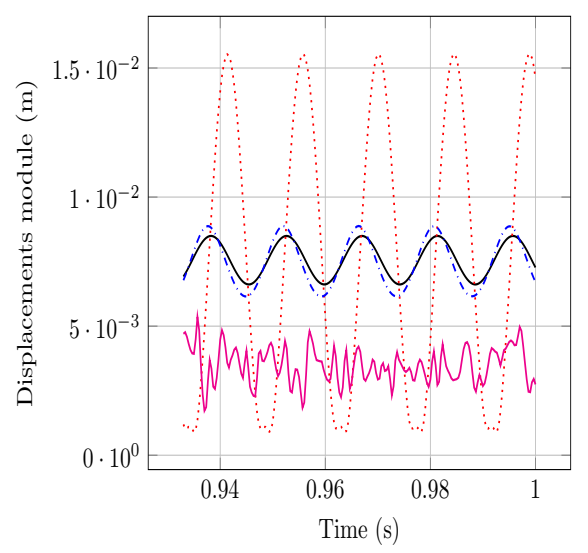

(b) $\Omega=2000 \mathrm{rpm}, \alpha_{f}=0.5$.

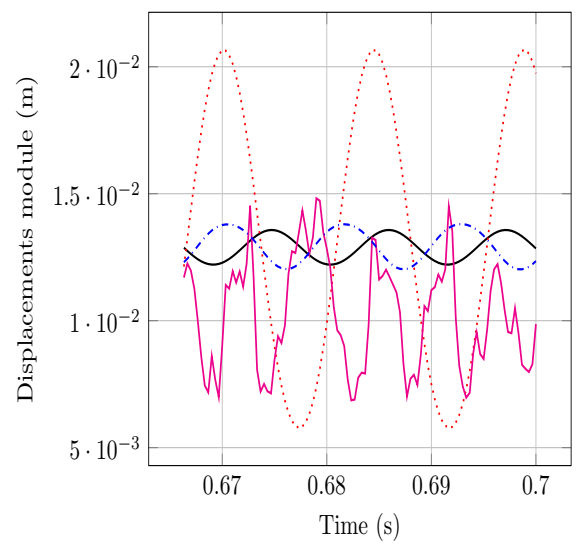

(c) $\Omega=4043 \mathrm{rpm}, \alpha_{f}=1.5$.

FIGURE 4. PERIODIC RESPONSE IN DISPLACEMENTS FOR THE LINEARISED MODE 1 RESONANT HARMONIC EXCITATION, LNM REDUCED BASIS

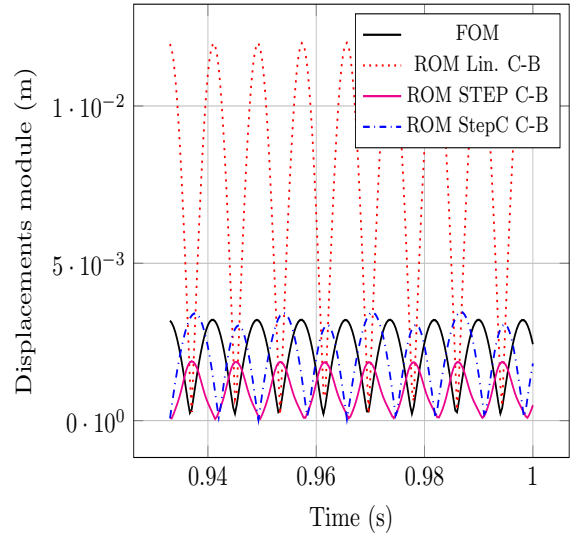

(a) $\Omega=0 \mathrm{rpm}, \alpha_{f}=0.5$.

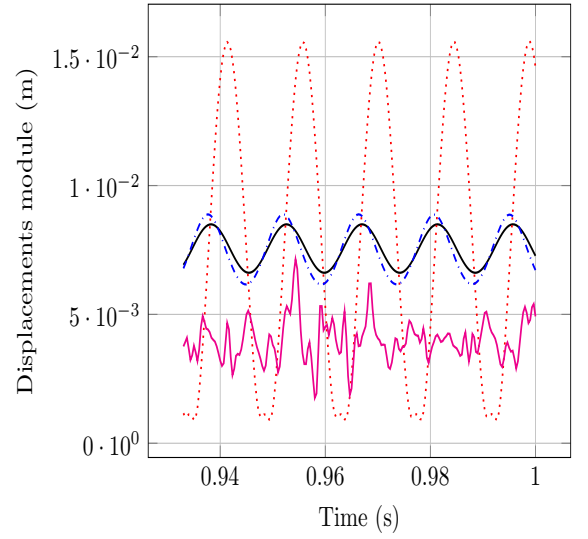

(b) $\Omega=2000 \mathrm{rpm}, \alpha_{f}=0.5$.

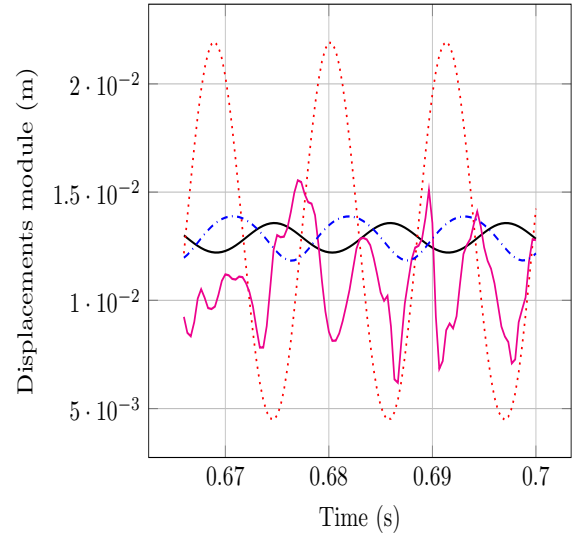

(c) $\Omega=4043 \mathrm{rpm}, \alpha_{f}=1.5$.

FIGURE 5. PERIODIC RESPONSE IN DISPLACEMENTS FOR THE LINEARISED MODE 1 RESONANT HARMONIC EXCITATION, CRAIG-BAMPTON REDUCED BASIS.

velocity for which the nonlinear force basis is constructed. However, as the accuracy is evaluated for the periodic solution, we only evaluate the accuracy in terms of response amplitude levels and frequency.

Error Analysis. The error of the ROMs periodic response is compared with respect to the FOM periodic response in terms of amplitude and response frequency. The POD based correction induces a time-shift in the response. Thus, the classical error computations cannot be carried out. In order to compare these solutions, the classical relative error is evaluated in the frequency domain. First, the periodic solution of the time-response is extracted, then, a Fast Fourier Transform (FFT) is carried out in order to transform the signals into frequency domain. Then, the error for each d.o.f. of the computed periodic response spectrum is performed as shown in Eqn. (20). 


$$
e_{r}(\%)=\frac{\left|u_{R O M}(\omega)-u_{F O M}(\omega)\right|}{\max \left\{u_{F O M}(\omega)\right\}} \cdot 100
$$

Table 1 presents the spectrum based maximum relative error at the control coordinate. For both reduced bases and all the rotating velocities the nonlinear StepC ROM provides more accurate results (up to 39 times more accurate) than the linearised ROM. The STEP method on the other hand provides better results than the linearised ROM in terms of amplitude, however, the shape of the solution presents high level of undesirable harmonics. For most cases the Craig-Bampton basis provides more accurate results than the LNM basis. The latter might be due to the presence of static deformations inside Craig-Bampton basis that provide a "knowledge" about the nonlinear behaviour of the structure in the reduced basis.

TABLE 1. SPECTRUM BASED RELATIVE ERROR (\%) OF THE REDUCED ORDER MODELS FOR THE LINEARISED MODE 1 RESONANT HARMONIC EXCITATION.

\begin{tabular}{lcccccc}
\hline & \multicolumn{2}{c}{$\Omega=0 \mathrm{rpm}$} & \multicolumn{2}{c}{$\Omega=2000 \mathrm{rpm}$} & \multicolumn{2}{c}{$\Omega=4043 \mathrm{rpm}$} \\
\hline Reduced basis & $\Phi_{L N M}$ & $\Phi_{C B}$ & $\Phi_{L N M}$ & $\Phi_{C B}$ & $\Phi_{L N M}$ & $\Phi_{C B}$ \\
\hline Linear & 268.74 & 272.70 & 40.51 & 40.68 & 28.41 & 25.43 \\
STEP & 50.51 & 53.25 & 54.2 & 47.23 & 20.49 & 15.88 \\
StepC & 11.84 & 8.97 & 2.64 & 2.58 & 2.09 & 3.29 \\
\hline
\end{tabular}

Forced response. The forced response of the structure for different excitation frequencies at a given rotation regime provides valuable information for the designer. For geometrically nonlinear structures, the value of the maximum amplitude and the resonant frequency may vary (Duffing effect). Furthermore, in the neighbourhood of the nonlinear resonance, the system can present more than one stable configuration for the same excitation frequency.

The forced response computed by the proposed ROMs are shown in Fig. 6 for 0 rpm, $2000 \mathrm{rpm}$ and $4043 \mathrm{rpm}$ rotating velocities. The solutions were computed for 120 excitation frequencies equally distributed between $55 \mathrm{~Hz}$ and $95 \mathrm{~Hz}$. In the present case, for each computation, the HHT- $\alpha$ time integration method was carried out, however, techniques like the Harmonic Balance Method (HBM) with continuation techniques properly capture the unstable solution.

It is observed that the nonlinear resonant frequency is shifted with respect to the linear one and that the nonlinear amplitude is smaller than the one obtained with the linearised ROM. For $\Omega=0 \mathrm{rpm}$ the displacements of the STEP model underestimates the displacements of the structure. The StepC model for all rotating velocities outside resonance provides the same results as the linearised ROM. 


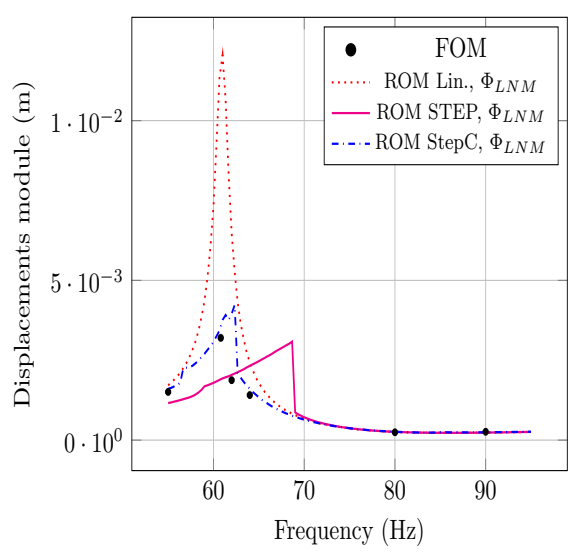

(a) $\Omega=0 \mathrm{rpm}, \alpha_{f}=0.5$.

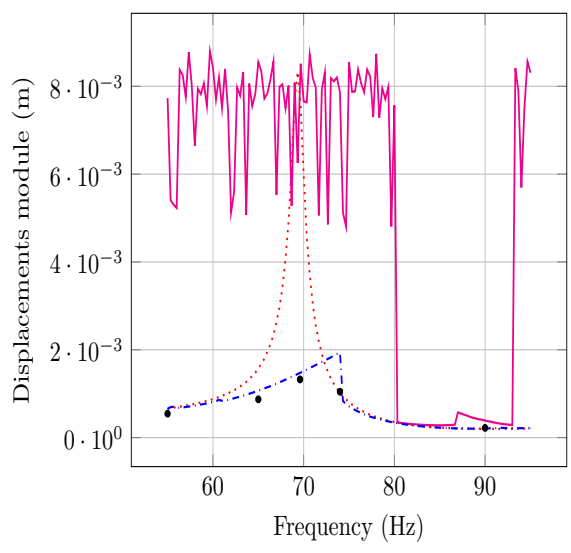

(b) $\Omega=2000 \mathrm{rpm}, \alpha_{f}=0.5$.

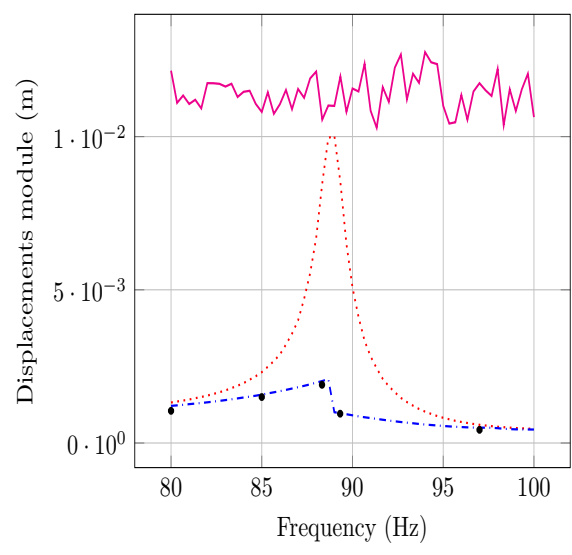

(c) $\Omega=4043 \mathrm{rpm}, \alpha_{f}=1.5$.

FIGURE 6. FORCED RESPONSE MODULE BETWEEN 55HZ AND 100 HZ FOR DIFFERENT ROTATING VELOCITIES.

TABLE 2. TIME PERFORMANCES OF THE ROMS.

\begin{tabular}{lcc}
\hline Model & Time & $\mathrm{t}_{F O M} / \mathrm{t}_{\text {ROM }}$ \\
\hline FOM & $90 \mathrm{~h} 52$ min $48 \mathrm{~s}$ & \\
ROM Lin. $\Phi_{L N M}$ & $15.2 \mathrm{~s}$ & 21524 \\
ROM STEP $\Phi_{L N M}$ & $19.6 \mathrm{~s}$ & 16692 \\
ROM StepC $\Phi_{L N M}$ & $19.8 \mathrm{~s}$ & 16523 \\
\hline
\end{tabular}

Time Performances. The time performances of the "ONline" stage between the ROMs and the FOM are evaluated here and shown in Tab. 2. The computational time of the ROMs is similar regardless the chosen reduced basis. Thus, only the time performances of the LNM with $\Omega=2000 \mathrm{rpm}$ and 3000 time steps are presented. Both ROM STEP and ROM StepC need the same computational "ONline" stage time to be solved. The Linear ROM is the quickest model with a 1.28 times factor with respect to nonlinear ROMs, however, the model is not precise enough. Then, the proposed StepC ROM provides the best compromise between accuracy and time consumption with a time gain of 16523 times quicker "ONline" stage than the FOM. Note that the FOM time consumption corresponds to a single CPU sequential time, the real clock time of the FOM corresponds to a lower value (about 5h) if computations are carried out with 25 cores in parallel. However, even if multicore computations are implemented the StepC ROM is about 1500 times quicker than the parallelised FOM.

With respect to the construction of the ROMs, in addition to the reduced basis computation, the "OFFline" stage comprises the evaluation of the polynomial coefficients $(\approx 540 \mathrm{~min}$ CPU time). Furthermore, to construct the nonlinear force basis of StepC nonlinear ROMs, an additional FOM computation is carried out for 60 excitation periods and 2000 time steps $(\approx 64 \mathrm{~h} \mathrm{CPU} \mathrm{time).}$ 


\section{CONCLUSIONS}

Reduced order models (ROMs) to study the dynamic response of geometrically nonlinear rotating structures are proposed here. The vibrations around the static equilibrium, induced by rotation, are supposed to be nonlinear enhancing the classical linearised approximation. The need to correctly represent the projection of the full order model (FOM) nonlinear forces in the reduced space is highlighted. A POD based correction is proposed to improve the accuracy of the reduced nonlinear forces and combined with a Craig-Bampton type reduced basis in order to study contact problem in the future. The proposed correction induces a time-shift in the displacements response of the nonlinear ROM. The latter forces are represented as a polynomial expansion by implementing the Stiffness Evaluation Procedure (STEP).

The proposed StepC ROM (STEP with nonlinear forces Correction Reduced Order Model) is evaluated for a blade application. The periodic solutions are obtained with the HHT- $\alpha$ time integration method. For all the studied loading cases the StepC ROM provides more accurate results than the linearised solution. The parametrised reduced basis produce accurate models for the studied rotating velocities. When the structure rotates, StepC ROM with Craig-Bampton reduced basis provides about 40 times more accurate results than the linearised ROM with an "ONline" stage about 1.26 times longer than the linearised ROM. However, the time performances with respect to the FOM are about 16500 times quicker to perform. The construction of the latter ROM is more expensive than the linearised ROM or than the STEP ROM as an additional nonlinear forces basis is computed. However, the improvements in accuracy and the validity make the model worthy to be built. Furthermore, for lower loading intensities, the accuracy of the ROM is conserved.

Thus, the proposed StepC model, is capable to provide better results than the classical Linear ROM for the studied resonant loading cases and rotating velocities providing the best compromise between the accuracy and the time construction of the ROM. Furthermore, the proposed StepC ROM is proven to be capable to reproduce the dynamics of a complex structure with industrial applications. In the near future, the proposed StepC correction with Craig-Bampton reduced basis will be enhanced to contact type nonlinearities by introducing explicit contact laws.

\section{ACKNOWLEDGMENT}

The authors thank R. Barrier at ONERA/DAAA for the aerodynamic design of the studied blade and A. Desanti at ONERA/DPI for developing the structural finite element model.

\section{REFERENCES}

[1] Chaturantabut, S., and Sorensen, D. C., 2009. "Discrete empirical interpolation for nonlinear model reduction". In Decision and Control, 2009 held jointly with the 2009 28th Chinese Control Conference. CDC/CCC 2009. Proceedings of the 48th IEEE Conference on, IEEE, pp. 4316-4321. 
[2] Ryckelynck, D., 2005. “A priori hyperreduction method: an adaptive approach”. Journal of computational physics, 202(1), pp. 346-366.

[3] Farhat, C., Chapman, T., and Avery, P., 2015. "Structure-preserving, stability, and accuracy properties of the energy-conserving sampling and weighting method for the hyper reduction of nonlinear finite element dynamic models". International Journal for Numerical Methods in Engineering, 102(5), pp. 1077-1110.

[4] Bond, B. N., and Daniel, L., 2007. "A piecewise-linear moment-matching approach to parameterized model-order reduction for highly nonlinear systems". IEEE Transactions on Computer-Aided Design of Integrated Circuits and Systems, 26(12), pp. 21162129.

[5] Rizzi, S. A., and Muravyov, A. A., 2001. "Improved equivalent linearization implementations using nonlinear stiffness evaluation". NASA/TM-2001-210838, L-18068, NAS 1.15:210838.

[6] Mignolet, M. P., Przekop, A., Rizzi, S. A., and Spottswood, S. M., 2013. "A review of indirect/non-intrusive reduced order modeling of nonlinear geometric structures". Journal of Sound and Vibration, 332(10), pp. 2437-2460.

[7] Capiez-Lernout, E., Soize, C., Akkaoui, Q., and Ohayon, R., 2017. "Uncertainty propagation in a nonlinear reduced-order model in internal elasto-acoustics”. In Congrès Français de Mécanique (CFM 2017), Vol. 199, Elsevier, pp. 1204-1209.

[8] Capiez-Lernout, E., Soize, C., and Mbaye, M., 2014. "Geometric nonlinear dynamic analysis of uncertain structures with cyclic symmetry-application to a mistuned industrial bladed disk". In International Conference on Uncertainty in Structural Dynamics, USD2014, pp. 1-14.

[9] Kim, E., and Cho, M., 2017. "Equivalent model construction for a non-linear dynamic system based on an element-wise stiffness evaluation procedure and reduced analysis of the equivalent system”. Computational Mechanics, 60(5), pp. 709-724.

[10] Thomas, O., Sénéchal, A., and Deü, J.-F., 2016. "Hardening/softening behavior and reduced order modeling of nonlinear vibrations of rotating cantilever beams". Nonlinear Dynamics, 86(2), Oct, pp. 1293-1318.

[11] Beley, J.-D., Shen, Z., Chouvion, B., and Thouverez, F., 2017. “Vibration non-linéaire de poutre en grande transformation". In Conference: CSMA 2017, At Giens, France.

[12] Huang, H., and Han, Q., 2010. "Research on nonlinear postbuckling of functionally graded cylindrical shells under radial loads". Composite Structures, 92(6), pp. 1352-1357.

[13] Zhang, J., Guo, L., Wu, H., Zhou, A., Hu, D., and Ren, J., 2014. "The influence of wind shear on vibration of geometrically nonlinear wind turbine blade under fluid-structure interaction”. Ocean engineering, 84, pp. 14-19.

[14] Sternchüss, A., 2009. "Multi-level parametric reduced models of rotating bladed disk assemblies". PhD thesis, Ecole Centrale Paris, France.

[15] Genta, G., and Silvagni, M., 2014. "On centrifugal softening in finite element method rotordynamics". Journal of Applied Mechanics, 81(1), p. 011001. 
[16] Craig, R., and Bampton, M., 1968. "Coupling of substructures for dynamic analyses”. AIAA journal, 6(7), pp. 1313-1319.

[17] Farhat, C., and Geradin, M., 1994. "On a component mode synthesis method and its application to incompatible substructures". Computers \& Structures, 51(5), pp. 459-473.

[18] Iwan, W. D., 1966. “A distributed-element model for hysteresis and its steady-state dynamic response". Journal of Applied Mechanics, 33(4), pp. 893-900.

[19] Brake, M. R., Groß, J., Lacayo, R. M., Salles, L., Schwingshackl, C. W., Reuß, P., and Armand, J., 2018. “Reduced order modeling of nonlinear structures with frictional interfaces”. In The Mechanics of Jointed Structures. Springer, pp. 427-450.

[20] Petrov, E., and Ewins, D., 2002. "Analytical formulation of friction interface elements for analysis of nonlinear multi-harmonic vibrations of bladed discs". In ASME Turbo Expo 2002: Power for Land, Sea, and Air, American Society of Mechanical Engineers, pp. 899-908.

[21] Petrov, E., Di Mare, L., Hennings, H., and Elliott, R., 2010. "Forced response of mistuned bladed disks in gas flow: A comparative study of predictions and full-scale experimental results". Journal of Engineering for Gas Turbines and Power, 132(5), p. 052504.

[22] Henry, R., 1981. “Contribution à l'étude dynamique des machines tournantes”. PhD thesis, INSA Lyon, France.

[23] Lülf, F. A., Tran, D.-M., Matthies, H. G., and Ohayon, R., 2015. "An integrated method for the transient solution of reduced order models of geometrically nonlinear structures". Computational Mechanics, 55(2), pp. 327-344.

[24] Balmaseda, M., Jacquet-Richardet, G., Placzek, A., and Tran, D.-M., 2018. "Reduced order models for dynamic analysis of nonlinear rotating structures". In Proceedings of 6th European Conference on Computational Mechanics / 7th European Conference on Computational Fluid Dynamics, Glasgow, UK.

[25] Heegaard, J.-H., and Curnier, A., 1993. "An augmented lagrangian method for discrete large-slip contact problems". International Journal for Numerical Methods in Engineering, 36(4), pp. 569-593.

[26] Chabrand, P., Dubois, F., and Raous, M., 1998. "Various numerical methods for solving unilateral contact problems with friction". Mathematical and Computer Modelling, 28(4-8), pp. 97-108.

[27] Sofonea, M., and Matei, A., 2012. Mathematical models in contact mechanics, Vol. 398. Cambridge University Press.

[28] Hong, S.-K., Epureanu, B. I., Castanier, M. P., and Gorsich, D. J., 2011. "Parametric reduced-order models for predicting the vibration response of complex structures with component damage and uncertainties". Journal of sound and vibration, 330(6), pp. 1091-1110.

[29] Kurstak, E., Wilber, R., and D’Souza, K., 2018. "Parametric reduced order models for bladed disks with mistuning and varying operational speed". Journal of Engineering for Gas Turbines and Power.

[30] Grolet, A., and Thouverez, F., 2010. "Vibration analysis of a nonlinear system with cyclic symmetry". Journal of Engineering for Gas Turbines and Power, 133(2), p. 022502.

[31] Hansen, P., 1990. "Truncated singular value decomposition solutions to discrete ill-posed problems with ill-determined numerical 
rank". SIAM Journal on Scientific and Statistical Computing, 11(3), pp. 503-518.

[32] Wiart, L., Atinault, O., Boniface, J.-C., and Barrier, R., 2016. "Aeropropulsive performance analysis of the NOVA configurations". 30th Congress of the International Council of the Aerodynamical Sciences.

[33] Wiart, L., Atinault, O., Grenon, R., Paluch, B., and Hue, D., 2015. "Development of nova aircraft configurations for large engine integration studies". In 33rd AIAA Applied Aerodynamics Conference, p. 2254.

[34] Cardarelli, F., 2000. Materials handbook. Springer. 